\title{
Research on Coordination Scheduling Model and Application for Parallel Production Lines
}

\author{
Hongguang Bo ${ }^{1, a}$, Longlong $\mathrm{Li}^{1, \mathrm{~b}}$, Yu Liu ${ }^{2,3}$, Jiaheng Wang ${ }^{1}$ \\ ${ }^{1}$ Faculty of Management and Economics, Dalian University of Technology, \\ Dalian 116024, China \\ ${ }^{2}$ CSR Qingdao Sifang Co., Ltd. Qingdao 266111, China \\ ${ }^{3}$ Crrc Central Research Institute Qingdao 266111, China \\ aemail: Lee_dlut@163.com, bemail:Ihfeng1201@163.com
}

Keywords: Parallel production line; Hybrid manufacturing process; Bi-level programming; Coordinating production scheduling

\begin{abstract}
For the order planning problem of parallel production lines in hybrid process manufacturing enterprises, a novel solving method of parallel production lines coordinating scheduling is developed based on bi-level programming theory. To maximize gross profit of unit output as upper level optimization objective, and minimum order processing procedure cost as lower level optimization objective, a bi-level programming model for coordinating production scheduling is established. A merge method of orders based on the feature mapping is presented to preprocess the orders which have multiple varieties and small batches. A solving method is developed based on GA, the model and algorithm is verified to be feasibility using the data of case enterprise.
\end{abstract}

\section{Introduction}

In the production practice of hybrid process manufacturing industry, there usually exist production lines with parallel processes. The parallel production lines show the production organization features of both flow shop and parallel machine manufacturing environment. The order planning problem of the parallel production lines is a kind of complex production scheduling optimization problem.

Many researches have been done to flow shop and parallel machine scheduling problem, and many valuable research results have been achieved. Fondrevelle [1] builds a replaceable production lines scheduling model of waiting time with upper and lower bound, and designs a branch and bound method solution for it. Chen[2] studying resource allocation problem under the parallel machine environment, develops a production scheduling model with the objective of the minimum total weighted completion time and minimum total weighted lag number of jobs, and comes up with a column generation-based branch and bound algorithm. Liu[3] presents a combination rule heuristic genetic algorithm for the scheduling problem with an objective of minimum makespan under the identical parallel machine environment. For the economic batch quantity scheduling problem in the flexible production line with unrelated parallel machines, Jenabi [4] designs two kinds of meta-heuristics algorithm of hybrid genetic algorithm(i.e. HGA) and simulated annealing algorithm(i.e. SA). Considering proportional job process time between stages, Sundararaghavan [5] builds an integer programming model for two-stage parallel production line scheduling problem, and a model algorithm combining heuristic rules and simulation is put forward.

For the order coordination scheduling problem of parallel production lines, by studying the bi-level decision model of order organizing in iron and steel group enterprise, a parallel production line coordination scheduling model of order oriented production group enterprise is built. We apply bi-level programming theory into the coordination between decision-making level and executive level. A feature attribute mapping-based order merge pretreatment method is developed. We also design to solve the GA of coordination scheduling bi-level programming model with parallel 
production lines.

\section{Problem Description}

In this paper, we take iron and steel enterprise for example. Iron and steel industry is a typical mixed flow type production industry which mainly selects and smelts black metal minerals. This kind of enterprise usually has multiple production bases with parallel manufacturing capacity, while the bases have multiple production lines with similar process flow.

The traditional production scheduling uses a serial production scheduling model, as put in Figure 1 part A. First, group enterprise completes the group-level rough balance plan of production scheduling and delivers it to each base. Then, the base completes the base-level fine balance plan of production scheduling. If any conflict is found in the plan, it will be fed back to group enterprise and applied to be adjusted.

Obviously, the serial production scheduling model means that repeated scheduling calculations are required to get the optimal scheduling result. Besides, group and base have slight difference on scheduling objectives: the group pays more attention to maximum of unit output gross profit in the view of entire enterprise, while the base pays more attention to complete the production tasks with a least possible cost. For this problem, a bi-level programming theory based parallel coordinating production scheduling model of multi-production lines in group enterprises is developed. Parallel coordinating production scheduling model is shown in figure 1 part $\mathrm{B}$.

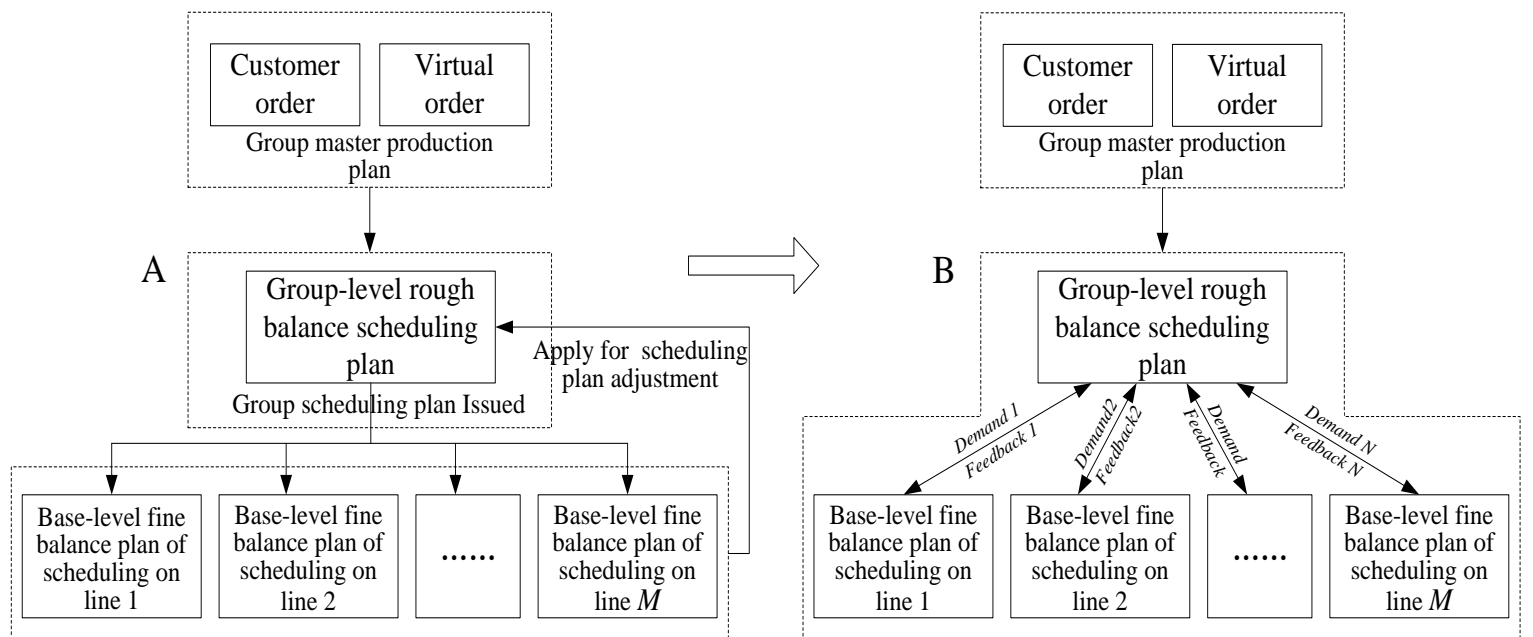

Figure.1. Change traditional serial production scheduling model into parallel coordinating production scheduling model

\section{Bi-level Programming Model}

\section{Model Establishment.}

We assume that an iron and steel group enterprise adopts the order oriented production mode. Group has $M$ production lines which are distributed in different regions and belong to different bases. All of them can produce multiple products. Production parallel scheduling problem of multi-production lines in this group can be mapped for a bi-level programming problem: the upper level is the group decision level, and the under level consists of $M$ structural -similar production lines. Each line contains I production processes and there are parallel machines for every process.

Model variables and decision variables of bi-level programming model are defined as follow:

Model variables:

$w_{(m, i)}^{(k, 0)}$ : order quantity of product $k$ on process $i$ of production line $m$;

$w_{(m, i)}^{(k, P)}$ : planning quantity of product $k$ on process $i$ of production line $m$;

$E_{(m, i)}:$ number of parallel machines on process $i$ of production line $m$; 
$T_{(m, i)}^{(j)}$ : available machine hours of machine $j$ on process $i$ during the plan period;

$\lambda_{(m, i)}^{(j, k)}$ : the reciprocal of yield of product $k$ on machine $j$ of process $i$ of production line $m$;

$K_{(m, i)}^{\text {OUT }}$ : number of varieties of finished products on process $i$ of production line m;

$K_{(m, i)}^{W I P}$ : number of varieties of products being processed on process $i$ of production line $m$;

$\tau_{(m, i)}^{(j, k)}$ : fixed man hour of product $k$ on process $i$ of production line $m$;

$r_{(m, i)}^{(k)}$ : unit price of product $k$ on process $i$ of production line $m$;

$c_{(m, i)}^{(k)}$ : unit cost of product $k$ on process $i$ of production line $m$;

Decision variables:

$x_{(m, i)}^{(j, k)}$ : order quantity of product $k$ on machine $j$ of process $i$ of production line $m$;

Thus, the total cost of the order arranged on process $i$ of production line $m$, is denoted as $C_{i}^{(m)}$ :

$$
C_{i}^{(m)}=\sum_{E_{(m, i)}} \sum_{K_{(m, i)}^{\text {OUT }}+K_{(m, i)}^{W I P}} C_{(m, i)}^{(k)} \cdot \lambda_{(m, i)}^{(j, k)} \cdot x_{(m, i)}^{(j, k)}
$$

The sum of finished products and products being processed in the entire group is denoted as $W_{(M, I)}^{(K, O+P)}$ :

$$
W_{(M, I)}^{(K, O+P)}=\sum_{M, I} \sum_{E_{(m, i)}} \sum_{K_{(m, i)}^{O U T}+K_{(m, i)}^{W I P}} \lambda_{(m, i)}^{(j, k)} \cdot x_{(m, i)}^{(j, k)}
$$

Unit gross profit during the plan period in the entire group is denoted as $R$ :

$$
R\left(x_{(m, i)}^{(j, k)}, C_{i}^{(m)}\right)=\left(\sum_{M, I} \sum_{E_{(m, i)}} \sum_{K_{(m, i)}^{\text {Out }}} r_{(m, i)}^{(k)} \cdot \lambda_{(m, i)}^{(j, k)} \cdot x_{(m, i)}^{(j, k)}-\sum_{M, I} C_{i}^{(m)}\right) / W_{(M, I)}^{(K, O+P)}
$$

Bi-level programming-based parallel line coordinating production scheduling model is built as follow:

$$
\begin{aligned}
& \text { Upper : } \quad \max R\left(x_{(m, i)}^{(j, k)}, C_{i}^{(m)}\right) \\
& \text { s.t. } \quad \sum_{M, I} \sum_{K_{(m, i)}^{\text {OUT }}} w_{(m, i)}^{(k, O)} \leq \sum_{M, I} \sum_{E_{(m, i)}} \sum_{K_{(m, i)}^{\text {OUI }}} \lambda_{(m, i)}^{(j, k)} \cdot x_{(m, i)}^{(j, k)} \leq \sum_{M, I} \sum_{K_{(m, i)}^{\text {OUT }}} w_{(m, i)}^{(k, P)} \\
& \text { Lower : } \quad \min C_{i}^{(m)} \\
& \text { s.t. } \quad \sum_{I} \sum_{K_{(m, i)}^{\text {OUT }}} w_{(m, i)}^{(k, O)} \leq \sum_{I} \sum_{E_{(m, i)}} \lambda_{(m, i)}^{(j, k)} \cdot x_{(m, i)}^{(j, k)} \leq \sum_{I} \sum_{K_{(m, i)}^{\text {OUT }}} w_{(m, i)}^{(k, P)} \\
& \lambda_{(m, i+1)}^{\left(j, k^{\prime \prime}\right)} \cdot x_{(m, i+1)}^{\left(j, k^{\prime \prime}\right)} \leq \lambda_{(m, i)}^{(j, k)} \cdot x_{(m, i)}^{(j, k)} \leq \lambda_{(m, i-1)}^{\left(j, k^{\prime}\right)} \cdot x_{(m, i-1)}^{\left(j, k^{\prime}\right)} \\
& \sum_{K_{(m, i)}^{\text {OUT }}+K_{(m, i)}^{W I P}} \tau_{(m, i)}^{(j, k)} \cdot \lambda_{(m, i)}^{(j, k)} \cdot x_{(m, i)}^{(j, k)} \leq T_{(m, i)}^{(j)} \\
& x_{(m, i)}^{(j, k)} \geq 0 \quad, \quad k, k^{\prime}, k^{\prime \prime}=1,2, \cdots, E_{(m, i)}
\end{aligned}
$$

Model Solution.

Order merger: We adopt the feature attribute-based product specification mapping method to carry out the order merger. After the mapping from the order demand feature attributes to the product specification, we can get the process and production organization features like machine norm and process route, and we can deal with the order merger according to the process route.

Order merger processing is shown in Figure 2. We take product BC-12Cr17 for example. 


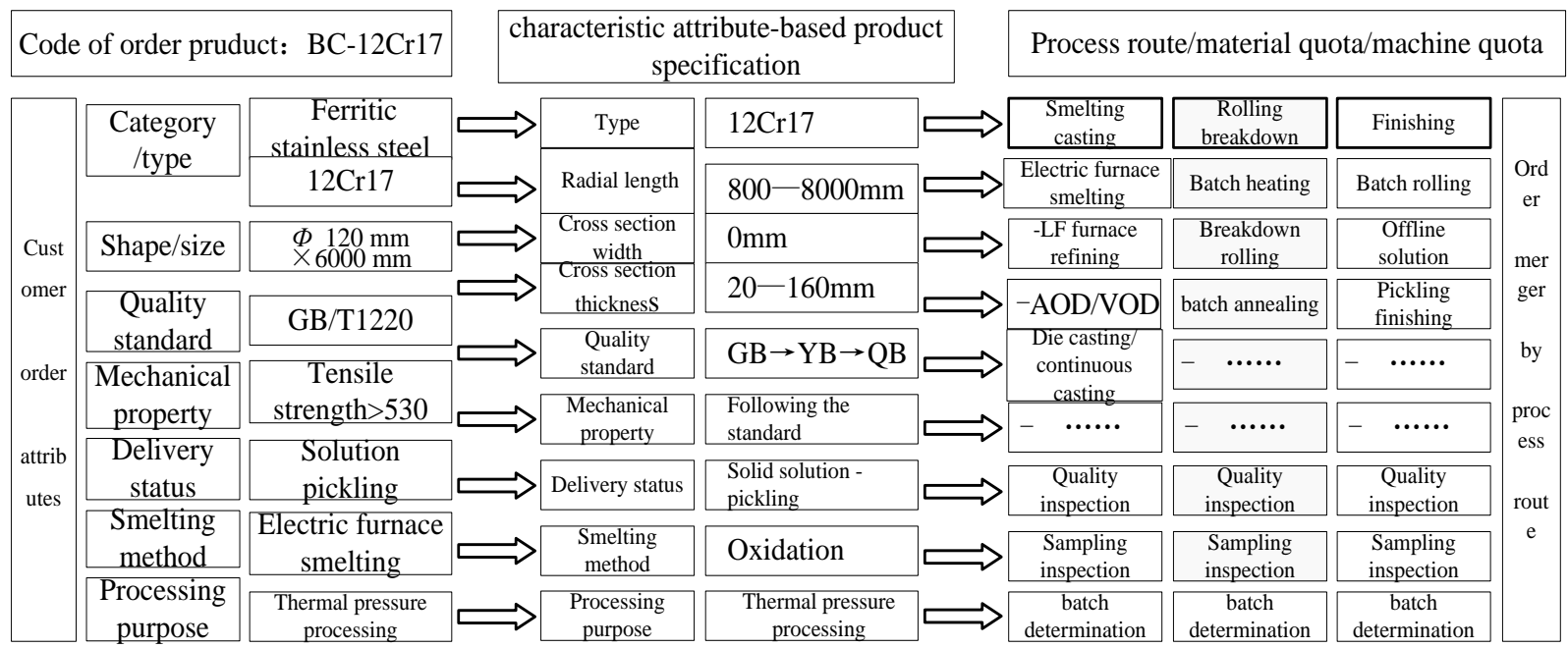

Figure.2. Feature attributes mapping-based order merger processing

Genetic algorithm: We adopt GA to solve the problem, and related definitions of GA go as follow:

Chromosome coding design: Using floating-point vector to represent the length of chromosome, which is the same to the upper level feasible solution vector $x_{(m, i)}^{(j, k)}$, i.e. $\operatorname{Crm}=\left\{x_{(m, i)[1]}^{(j, k)}, x_{(m, i)[2]}^{(j, k)}, \cdots, x_{(m, i)[W]}^{(j, k)}\right\}$;

Population initialization: Generating a group of chromosomes randomly (with a population size of $W$ ), establishing the initial population with the chromosomes under the restricts (5);

Lower level feedback: Lower level taking initialized chromosome $x_{(m, i)}^{(j, k)}$ of Upper level as parameter, solving the minimum value of problem $C_{i}^{(m)}$, feeding back this value to the objective $\max R\left(x_{(m, i)}^{(j, k)}, C_{i}^{(m)}\right)$ of Upper level;

Fitness function: Taking $\max R\left(x_{(m, i)}^{(j, k)}, C_{i}^{(m)}\right)$ of Upper level as fitness function;

Selecting operation of GA: Adopting the regular geometric sorting method, $P[\mathrm{i}]=\rho \cdot(1-\eta)^{r-1}$, in which $\eta$ is the selecting probability of the best individual, $r$ is the sorting of individual fitness, $\rho=\eta /\left(1-(1-\eta)^{W}\right)$;

Crossover of GA: Adopting the convex crossover of arithmetic crossover, selecting chromosome to crossover with a probability of $P$;

Mutation of GA: Selecting chromosome individual $x_{(m, i)}^{(j, k)}$ randomly, letting it be non-uniform distribution random number: when $\mathrm{ra} \geq \mathrm{rs} ; \quad x_{(m, i)}^{(j, k)}=x_{(m, i)}^{(j, k)}+\left(\theta^{x}-x_{(m, i)}^{(j, k)}\right) \cdot f(g) ; \quad$ when $\quad \mathrm{rb}<\mathrm{rs}$; $x_{(m, i)}^{(j, k)}=x_{(m, i)}^{(j, k)}+\left(x_{(m, i)}^{(j, k)}-\theta_{x}\right) \cdot f(g)$.

$\mathrm{ra}, \mathrm{rb}$, rs are uniformly distributed random numbers of interval $(0,1), \boldsymbol{\theta}^{x}$ and $\boldsymbol{\theta}_{x}$ are the upper and lower bounds of $x_{(m, i)}^{(j, k)}, f(g)=\left(r_{b} \cdot(1-g / G)\right)^{\omega}, g$ and $G$ are the generation with the largest sum, $\omega$ is the accelerating factor.

\section{Experimental Study}

We take an iron and steel group enterprise for example to conduct the experimental study of model and algorithm in this paper. This enterprise has 3 parallel integration production lines of steelmaking - continuous casting and hot rolling which belong to different bases.

We choose a set of orders received at July 2011 to schedule. The order includes bearing steel, 
stainless steel and alloy steel, more than 30 kinds of round steel and wire rod steel products. With features attribute-based product specification mapping method, we first merge pretreatment on orders to form the group to-be-scheduled order list. After the necessary standardization, a to-be-scheduled order list is formed as in table 1.

Table.1. Product information of to-be-scheduled order

\begin{tabular}{|c|c|c|c|c|c|c|}
\hline Product & $\begin{array}{l}\text { Bearing steel } \\
\text { round bar } \\
\Phi 50-200 \mathrm{~mm}\end{array}$ & $\begin{array}{l}\text { Bearing steel } \\
\text { wire rod } \\
\Phi 5.5-40 \mathrm{~mm}\end{array}$ & $\begin{array}{l}\text { Stainless steel } \\
\text { round bar } \\
\Phi 40-180 \mathrm{~mm}\end{array}$ & $\begin{array}{l}\text { Stainless steel } \\
\text { wire rod } \\
\Phi 10-30 \mathrm{~mm}\end{array}$ & $\begin{array}{l}\text { Alloy steel } \\
\text { round bar } \\
\Phi 10-60 \mathrm{~mm}\end{array}$ & $\begin{array}{l}\text { Alloy steel } \\
\text { wire rod } \\
\Phi 1.0-18 \mathrm{~mm}\end{array}$ \\
\hline $\begin{array}{l}\text { Order } \\
\text { quantity }\end{array}$ & 30.3 & 26.9 & 16.6 & 11.9 & 12.3 & 10.8 \\
\hline Plan qutity & 31.1 & 28.1 & 18.1 & 12.5 & 13.2 & 12.9 \\
\hline Unit price & 0.49 & 0.51 & 0.53 & 0.55 & 0.57 & 0.63 \\
\hline $\begin{array}{l}\text { Process } \\
\text { route }\end{array}$ & $P_{0}^{(1)} \rightarrow P_{1}^{(1)}$ & $P_{0}^{(1)} \rightarrow P_{1}^{(1)} \rightarrow P_{2}^{(1)}$ & $P_{0}^{(2)} \rightarrow P_{1}^{(2)}$ & $P_{0}^{(2)} \rightarrow P_{1}^{(2)} \rightarrow P_{2}^{(2)}$ & $P_{0}^{(3)} \rightarrow P_{1}^{(3)} \rightarrow P_{2}^{(3)}$ & $\begin{aligned} & P_{0}^{(3)} \rightarrow P_{1}^{(3)} \\
\rightarrow & P_{2}^{(3)} \rightarrow P_{3}^{(3)}\end{aligned}$ \\
\hline $\begin{array}{l}\text { Alternative } \\
\text { process } \\
\text { route } 1\end{array}$ & $P_{0}^{(3)} \rightarrow P_{1}^{(3)}$ & $P_{0}^{(3)} \rightarrow P_{1}^{(3)} \rightarrow P_{2}^{(3)}$ & $P_{0}^{(1)} \rightarrow P_{1}^{(1)}$ & $P_{0}^{(1)} \rightarrow P_{1}^{(1)} \rightarrow P_{2}^{(1)}$ & $P_{0}^{(1)} \rightarrow P_{1}^{(1)} \rightarrow P_{2}^{(1)}$ & 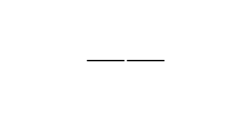 \\
\hline $\begin{array}{l}\text { Alternative } \\
\text { process } \\
\text { route } 2\end{array}$ & $P_{0}^{(2)} \rightarrow P_{1}^{(2)}$ & $P_{0}^{(2)} \rightarrow P_{1}^{(2)} \rightarrow P_{2}^{(2)}$ & $P_{0}^{(3)} \rightarrow P_{1}^{(3)}$ & $P_{0}^{(3)} \rightarrow P_{1}^{(3)} \rightarrow P_{2}^{(3)}$ & $P_{0}^{(2)} \rightarrow P_{1}^{(2)} \rightarrow P_{2}^{(2)}$ & - \\
\hline
\end{tabular}

Solve the parallel lines order scheduling problem with the model and algorithm above. Set the population size as $W=30$; selecting probability of the best individual as $\eta=0.07$, probability of crossover as $p_{c}=0.96$; maximum number of iterations as $G=300$. Result of parallel lines order scheduling is shown in Table 2.

Table.2. Result of parallel lines order scheduling

\begin{tabular}{|c|c|c|c|c|c|c|c|c|c|c|}
\hline \multirow[t]{2}{*}{ Parallel lines } & \multicolumn{3}{|c|}{$\begin{array}{c}\Phi 750 \\
\text { blooming-continuous } \\
\text { rolling lines }\end{array}$} & \multicolumn{3}{|c|}{$\begin{array}{c}\Phi 650 \\
\text { blooming-continuous } \\
\text { rolling lines }\end{array}$} & \multicolumn{4}{|c|}{$\begin{array}{l}\Phi 850 \\
\text { blooming-continuous rolling } \\
\text { lines } \\
\end{array}$} \\
\hline & $P_{0}^{(1)} \rightarrow$ & $P_{1}^{(1)}$ & $\rightarrow P_{2}^{(1)}$ & $P_{0}^{(2)} \rightarrow$ & $P_{1}^{(2)}$ & $\rightarrow P_{2}^{(2)}$ & $P_{0}^{(3)}$ & $\rightarrow P_{1}^{(3)}$ & $\rightarrow P_{2}^{(3)}$ & $\rightarrow P_{3}^{(3)}$ \\
\hline $\begin{array}{l}\text { Finished } \\
\text { product } \\
\text { quantity }\end{array}$ & & 18.62 & 18.69 & & 16.17 & 18.28 & & 12.11 & 14.13 & 10.80 \\
\hline $\begin{array}{l}\text { Quantity of } \\
\text { product being } \\
\text { processed }\end{array}$ & 47.80 & 20.10 & & 46.18 & 20.31 & & 47.26 & 27.59 & 11.25 & \\
\hline $\begin{array}{l}\text { Sum of } \\
\text { finished } \\
\text { products }\end{array}$ & & & 7.31 & & & 34.45 & & & 37.04 & \\
\hline $\begin{array}{l}\text { Sum of } \\
\text { products } \\
\text { being } \\
\text { processed }\end{array}$ & 67.5 & & & 66. & & & & 86.10 & & \\
\hline Unit cost & & 0.39 & 0.46 & & 0.43 & 0.53 & & 0.40 & 0.47 & 0.51 \\
\hline $\begin{array}{c}\text { Gross profit of } \\
\text { unit steel }\end{array}$ & & total sa & ales reven & $\begin{array}{r}\text { e - total } \\
(57.72\end{array}$ & $\begin{array}{l}\text { produ } \\
-49.53\end{array}$ & $\begin{array}{l}\text { etion cos } \\
\text { 3) } / 108.8\end{array}$ & $\begin{aligned} & / \text { total } \\
= & 0.075\end{aligned}$ & order pr & oduction & $n=$ \\
\hline
\end{tabular}

As shown in Table 2, gross profit of per ton steel about this set of order is about 0.075 , and the gross profit rate is about $14.19 \%$. The production cost of per ton steel of the base lines are is kept on a low level. 


\section{Conclusion}

Parallel lines order scheduling problem has the features of both flow shop and parallel machine problem. For the multi-production lines coordination scheduling problem of order oriented production group enterprise, a bi-level programming theory based parallel lines coordinating scheduling model is developed. In terms of model solving, a feature attributes mapping based order merger method is put forward. A GA is designed to solve the model. In the end, an experimental study is conducted using the business data from the case enterprise to confirm the model and the algorithm. The result has shown that the model properly solves the order optimal allocation and cost control problem. The equilibrium of capacity and resource allocation is achieved. It also supports the enterprise production and marketing integration.

\section{Acknowledgement}

This work was supported by the National Science and Technology Support Program (2015BAF08B02), the National Natural Science Foundation of China (71172137), and the Fundamental Research Funds for the Central Universities (DUT14RW101)

\section{References}

[1] Fondrevelle J, Oulamara A, Portmann M C. Permutation flowshop scheduling problems with maximal and minimal time lags[J]. Computers \& Operations Research, 2006(33):1540-1556.

[2] Chen Z L. Simultaneous job scheduling and resource allocation on parallel machines[J]. Annals of Operations Research, 2004, 129(1):135-153.

[3] Liu M, Wu C, Yang Y J. Genetic algorithm method based on combinatorial rules in identical parallel machine scheduling problem[J]. Acta Electronica Sinica, 2000, 28(5): 52-54.

[4] Jenabi M, Fatemi Ghomi S M T, Torabi S A, Karimi B. Two hybrid meta-heuristics for the finite horizon ELSP in flexible flow lines with unrelated parallel machines[J]. Applied Mathematics and Computation.2007, 186(1): 230-245.

[5] Sundararaghavan P, Kunnathur A, Viswanathan I. Minimizing make span in parallel flow shop[J]. The Journal of the Operational Research Society, 1997, 48: 834-842. 